\title{
Towards a general theory of immunity?
}

Gérard Eberl ${ }^{1}$ and Thomas Pradeu ${ }^{2}$

\section{Forthcoming in Trends in Immunology}

\author{
${ }^{1}$ Institut Pasteur, Microenvironment \& Immunity Unit, 75724 Paris, France \\ ${ }^{1}$ INSERM U1224, 75724 Paris, France \\ ${ }^{2}$ ImmunoConcept, UMR5164, CNRS and University of Bordeaux, 33076 Bordeaux, \\ France
}

Correspondence: gerard.eberl@pasteur.fr and thomas.pradeu@u-bordeaux.fr

\begin{abstract}
Theories are indispensable to organize immunological data into coherent, explanatory, and predictive frameworks. Here we propose to combine different models to develop a unifying theory of immunity, which situates immunology in the wider context of physiology. We believe that the immune system will be increasingly understood as a central component of a network of partner physiological systems that connect to maintain homeostasis.
\end{abstract}

\section{Why a theory of immunity?}

Since its inception in the late 19th century, immunology has been embedded into medicine, aiming to explain how we do not succumb to infectious microbes. Immunology is practical: based on our understanding of the immune system, we have developed vaccines and immunotherapies against infection and cancer, and are countering its destructive effects during pathological inflammation. Nonetheless, immunology was awarded several Nobel Prizes for its theories, in 1908 to Metchnikoff and Ehrlich for their complementary frameworks ("cellular" and "humoral"), in 1960 to Burnet for founding concepts on tolerance and the clonal selection theory, in 1984 to Jerne for theories "concerning the specificity in development and control of the immune system". These awards underscore the fundamental challenge in explaining how the immune system recognizes pathogens. 
Immunological theories allowed designing experiments to advance our understanding of these mechanisms, and develop tools to translate this understanding into medicine.

\section{Why a new theory?}

The self-non-self theory has dominated immunology ever since it was proposed by Burnet in the 1940s, drawing the line between us and infectious microbes by excluding all microbes. The Danger model, developed by Matzinger in the 1990s, proposes however that the immune system is not activated by non-self, but rather by the damage that microbes, or anything else, inflicts to our cells and tissues [1]. Nevertheless, microbial products, such as LPS, can activate the immune system without damaging cells and tissues (even though it may be viewed as a proxy of danger). Therefore, Jaeger, Vivier and one of us proposed in 2013 that the immune system is not fundamentally activated by non-self or danger, but rather by a change in normality, termed "discontinuity": the rate of change determines to the amplitude of effector responses [2,3]. Of note, discontinuities come in many types that define the types of immune response, and include anything from incoming microbes, chemicals, damaged extracellular matrix, different modes of cell death or increased expression of self-proteins. This theory generalizes the function of immunity to the maintenance of homeostasis, rather than only to defense against pathogens, a notion discussed previously by McFall-Ngai [4] and by one of us [5] in the context of the evolution of microbiota-host interactions. Immunity may be broadened to include tissue, cell and molecular repair, as proposed in 2016 by Kourilsky [6]. So what is now missing to understand immunity?

\section{The equilibrium model of immunity}

These theories explain how the immune system is activated, but do not account for more general levels of immune cross-regulations. Immune responses are induced by discontinuities but, importantly, they come into several mutually inhibitory types [7]. Type 1 responses are directed against infected or transformed cells, induced by mechanisms that detect intracellular discontinuities, such as the MHC class I antigen processing pathway and cytoplasmic pattern recognition receptors (PRRs). Type 3 responses, in contrast, are induced by discontinuities that affect the extracellular space, such as bacterial or fungal infections, and typically involve phagocytes. In the case of large extracellular threats, such as helminthes, which cannot be eliminated by 
phagocytes, type 2 responses are induced that use tissue repair processes to block entry of the parasites into tissues. Mucus production and collagen deposition are characteristic of such responses, which may lead to tissue fibrosis if prolonged. A fourth type of response is proposed that prevents microbes from approaching sensitive tissues where they would trigger damaging inflammation, such as the eye or the intestinal lamina propria [8].

As we now realize, all these responses are active in a healthy organism, as viruses, bacteria, fungi and parasites are ever present in the microenvironment at mucosal surfaces and within, and tissues have to be constantly repaired. We also know that the different types of immune responses, including regulatory $\mathrm{T}$ cells (Tregs), are mutually inhibitory, a cross-regulation that defines a homeostatic equilibrium. An ongoing anti-helminth type 2 response, for example, blocks type 1 anti-viral responses or type 3 anti-bacterial responses [9]. Conversely, the antibioticmediated destruction of bacteria leads to decreased type 3 responses, and thus to increased susceptibility to allergy and improved anti-viral responses [10].

\section{Immunity, physiology and ecology}

The immune system is embedded in an organism that has limited resources [11]. We all have experienced the exhausting effects of an infection, the immunity to which requires at times massive mobilization of energy. Conversely, stress can divert energy away from the immune system to accomplish an urgent task, such as the fight or flight response to a predator. Thus, the immune system is dependent on the availability of resources stored in cells and specialized tissues, and on resource allocation centrally managed, in many animals, by the brain. The nervous system, in its own way, perceives discontinuities within the organism and in the environment, and coordinates behavioral and physiological processes to maintain homeostasis [12], and thus, survival of the organism. As emphasized recently by Veiga-Fernandes and Freitas, the immune and the nervous systems are comparable in that both sense perturbations in the organism and in its environment [13], and interact intimately through neuroimmune cells "units" [14].

\section{Towards a general and practical theory of immunity}

In conclusion, in order to best describe the functioning of the immune system, a general theory of immunity has to take into account at least 3 levels of activation and 
regulation that orchestrate homeostasis of the organism (Figure 1): 1) the activation of the immune system by discontinuities induced by microbes, tumors, and injuries, 2) the mutual regulation of immune responses to distinct types of discontinuities, and 3) the global regulation of the immune system within the organism. While most of $20^{\text {th }}$ century immunology has focused on understanding level 1, immunology now integrates microbiota and tissue repair as essential components of its reactivity (level 2). Level 3 is progressively opening up, as physiology and neurosciences develop powerful new experimental tools, while immunologists become aware of the fundamentally transversal nature of their field [15] (Textbox). If the perspective presented here is correct, we can predict that immunology will be increasingly understood as a component of a large network of partner biological domains (in particular endocrinology, neurosciences, the study of metabolism, the study of repairregeneration), in which immunology is likely to play a central role.

\section{References}

1 Matzinger, P. (1994) Tolerance, danger, and the extended family. Annu Rev Immunol 12, 991-1045

2 Pradeu, T. et al. (2013) The speed of change: towards a discontinuity theory of immunity? Nat Rev Immunol 13 (10), 764-769

3 Pradeu, T. and Vivier, E. (2016) The discontinuity theory of immunity. Sci. Immunol. 1, aag0479

4 McFall-Ngai, M. (2007) Adaptive immunity: care for the community. Nature 445 (7124), 153

5 Eberl, G. (2010) A new vision of immunity: homeostasis of the superorganism. Mucosal Immunol 3, 450-460

6 Kourilsky, P. (2016) The natural defense system and the normative self model. F1000Research 5, 797

7 Eberl, G. (2016) Immunity by Equilibrium. Nat Rev Immunol 16, 524-532

8 Matzinger, P. and Kamala, T. (2011) Tissue-based class control: the other side of tolerance. Nat Rev Immunol 11 (3), 221-230

9 Osborne, L.C. et al. (2014) Coinfection. Virus-helminth coinfection reveals a microbiota-independent mechanism of immunomodulation. Science 345 (6196), 578-582

10 Baldridge, M.T. et al. (2015) Commensal microbes and interferon-lambda determine persistence of enteric murine norovirus infection. Science 347 (6219), 266-269

11 Schulenburg, H. et al. (2009) Introduction. Ecological immunology. Philosophical transactions of the Royal Society of London. Series B, Biological sciences 364 (1513), 3-14

12 Kotas, M.E. and Medzhitov, R. (2015) Homeostasis, inflammation, and disease susceptibility. Cell 160 (5), 816-827

13 Veiga-Fernandes, H. and Freitas, A.A. (2017) The S(c)ensory Immune System Theory. Trends Immunol 
14 Veiga-Fernandes, H. and Mucida, D. (2016) Neuro-Immune Interactions at Barrier Surfaces. Cell 165 (4), 801-811

15 Qiu, Y. et al. (2014) Eosinophils and type 2 cytokine signaling in macrophages orchestrate development of functional beige fat. Cell 157 (6), 1292-1308

\section{Textbox: Assessing the physiological integration of the immune system}

Integration of the immune system with the other systems in the organism (see Figure 1, level 3) is necessary to maintain homeostasis. All systems cross-talk to generate energy, growth, movement and defense, while keeping life parameters within physiological levels. We now come to realize that the immune system is key to the function of the other systems and in turn, requires input from these other systems to function. Therefore, a more holistic approach to immunology appears necessary to develop a full understanding of the immune system. Here are a few examples of physiological cross-regulations with the immune system that are coming to the forefront of research, and carry much potential for discovery and progress, both in the lab and in the clinic.

1. Nervous system: the cross-talk between the nervous and immune systems has been investigated for decades, but novel technologies to manipulate both molecules and cells with exquisite precision in both systems, such as optogenetics, allow for detailed mechanistic investigations of nervous and immune networks (Gradinaru, V. et al., Science 2009). Recent advances show how immune cells and neurons interact during health and disease (Kipnis, J., Science 2016), but the regulation of cognition by immunity, and immunity by cognition, remain intriguing cross-talks to explore.

2. Digestive system: the role of the intestinal microbiota in digestion, of its metabolites in the immune system and hepatic functions, the regulation of the microbiota and hepatic functions by the immune system, and, more generally, the cross-talk between metabolism and immunity, rise profound interest in the research community, as perversion of these cross-talks are associated with metabolic diseases such as diabetes (Ghesquiere, B. et al., Nature 2014). Digestion, metabolism and immune reactions are also linked as sources and sink of energy (O'Neill, L.A. et al., Nat Rev Immunol 2016). In animals, the management of energy at the organismal 
level requires the nervous system to integrate other energetic priorities, such as movements generated by the skeletal system, itself under the control of perception by the CNS. Thus, exploring energy management requires many systems to be investigated as integrated networks.

3. Skeletal and circulatory systems: bones, muscles and vessels are prone to injury, and must be repaired. Type 2 immune responses co-opt repair mechanisms to fight large parasites or develop allergic reactions (Gause, W.C. et al., Nat Rev Immunol 2013). They also play a central role in tissue repair, the deregulation of which leads to fibrosis and vascular diseases (Gieseck, R.L., et al., Nat Rev Immunol 2017). It remains however unclear to what extent tissue repair processes are dependent on type 2 responses, and to what extent repair processes have shaped pro-allergic and antihelminth responses.

4. Endocrine system: hormones mediating stress, sex, energy partition or circadian rhythms all have effects on immune cells, involving direct effects of nuclear hormone receptors in the regulation of immune genes (Glass, C.K. and Ogawa, S., Nat Rev Immunol 2006). Less clear in many cases is the logic of such regulation at the organismal level, and the effect of the immune system on these processes that control physiological homeostasis during adaptation to the environment.

5. Aging: with time, integration of the immune system may become deregulated as its cross-talk with other systems may suffer from the senescence of stem cells and the consequent loss of regenerative capacity in its own cells and tissues, as well as in cells and tissues of the other systems (Oh, J., Nat Med 2014). Such loss of integration may favor the development of chronic inflammatory pathologies that are characteristic of old age. 


\section{Figure:}
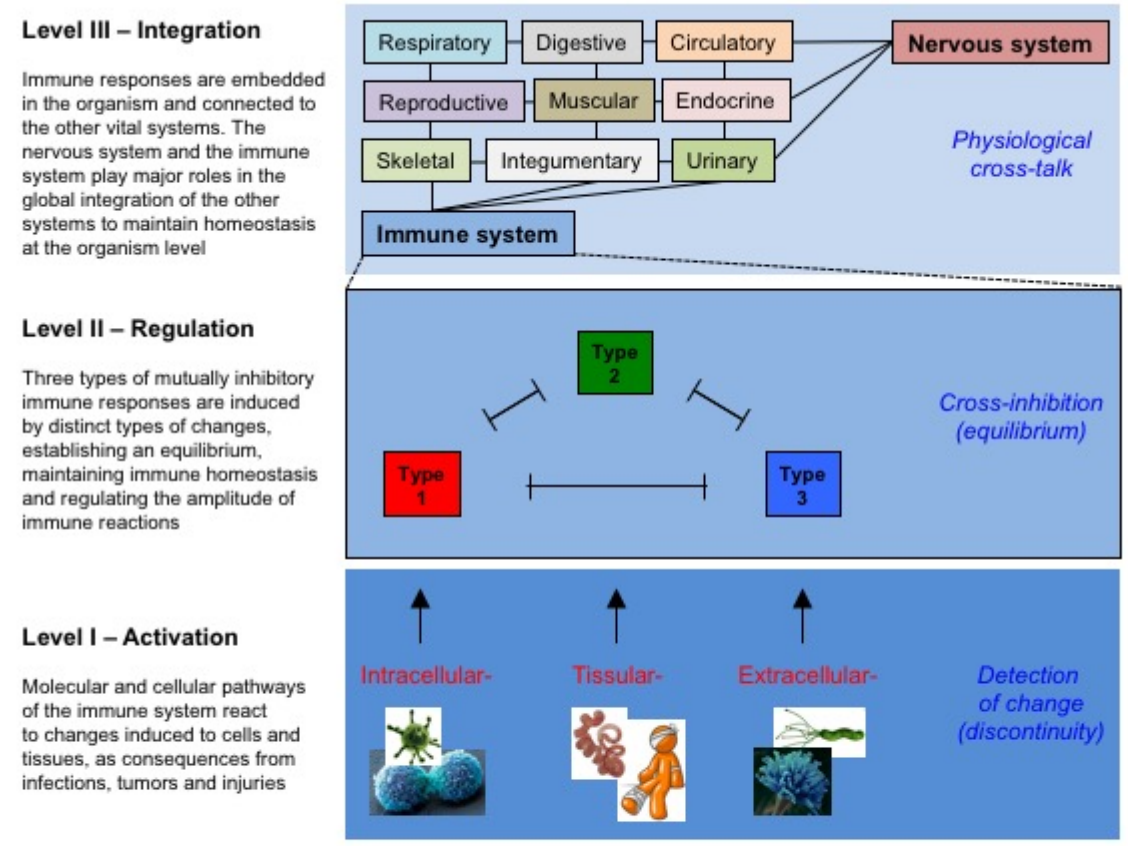

Figure 1. The immune system, as any other system, functions at different levels (or scales) in order to maintain homeostasis of the organism. As a consequence, immune responses are regulated, by different mechanisms, at each of these levels. 